\title{
ERROR ESTIMATES FOR ASYMPTOTIC REPRESENTATIONS OF INTEGRALS
}

\author{
J. J. MAHONY
}

(Received 3 October 1969, revised 12th June 1970)

Communicated by A. F. Pillow

\begin{abstract}
For large real positive values of the parameter $k$ asymptotic representations of integrals of the form$$
\int_{a}^{b} \exp \{k g(u)\} f(u) d u,
$$

where $f$ and $g$ are analytic functions, can be obtained by using methods such as steepest descents. Here methods are considered for obtaining estimates, for fixed values of $k$, of the minimum errors achievable when such asymptotic representations are appropriately curtailed. A priori criteria are derived for the optimum point at which to curtail such asymptotic representations. Both the curtailment points and the minimum errors are related to the distance between certain marked points on the path of integration and the singular points of $f(u)$ and the zeros of $g(u)$. The analysis permits the determination of errors whose presence is not indicated by the numerical behaviour of the asymptotic representations. It is also capable of extension to complex parameters $k$ and to the derivation of asymptotic representations for the most significant errors. It can therefore be used to extend the domain of $k$ for which asymptotic representations are available.
\end{abstract}

\section{Introduction}

There are various techniques available for obtaining asymptotic representations of integrals whose integrands involve a complex exponential function containing a large parameter. However, in many cases, error estimates for these asymptotic representations are not available even when it can be established that the series part is asymptotic in the sense of Poincaré. Problems can arise from physical contexts in which there is considerable interest in the mathematical nature and numerical magnitude of the error terms involved in the use of asymptotic representation of such integrals. An example of this arose in some recent work of the author [1] on the reflection of short waves by a variable medium. In that problem the reflected wave, the determination of amplitude of which was the primary aim of the investigation, occurs only in the transcendentally small error term when the integrals involved are approximated by standard means. Mathematically this implies that the reflection coefficient is small in comparison with any negative power of the large parameter involved in the limit as this parameter tends to infinity. Practically one wishes to make use of the asymptotic representations for finite, and often only moderately large, values of the parameter. In such cases 
the limit statement is not helpful. What is required is an estimate of the error for a given large value of the parameter.

In a recent article Olver [2] has drawn attention to the serious errors which may result when asymptotic representations are used to give numerical results. The distressing feature of the example quoted by Olver is the lack of indication from the numbers that the results are seriously in error. Thus the usual trust that is placed in asymptotic series whose diminution rate is sufficiently good is not always well-founded. This paper describes an initial attempt to investigate the factors which control the error size and hence to determine the most significant error which arises in the determination of certain classes of asymptotic representations for fixed values of the large parameter.

Here interest will be centred on integrals of the form

$$
\int_{a}^{b} \exp [k g(u)] f(u) d u,
$$

where $g$ and $f$ are holomorphic functions in the whole complex plane save for poles and branch lines and $k$ is a parameter, taken here to be real, large positive. It would appear that analytic continuation arguments will permit extension of the results to complex values of $k$ but this adds excessive complication to the analysis for a preliminary investigation. Such integrals are normally estimated by one of the related methods of steepest descents or stationary phase which lead to standard asymptotic representations. What is of concern here is the nature of the errors of the asymptotic representations obtained in this way. There are far too many cases to justify a general discussion of such integrals so that only a few particular examples, hopefully chosen to be representative of the analysis likely to be required, are considered. Throughout emphasis is placed on methods for determining the general form of the error terms and looking at types of analysis which are likely to be required. No attempt has therefore been made to derive complete answers for the examples under consideration once the problem has been reduced to a form amenable to standard techniques. The present investigation does appear to provide a reasonable guide as to what would be involved in any complete investigation of a specific example.

\section{Reflected wave integral}

The first example considered is

$$
F(x, k)=\int_{x}^{\infty} e^{i k u} f(u) d u
$$

which is of the type which arises in the reflection of short waves from a variable medium (Mahony [1]). It will be assumed that $f(u)$ has no poles on, nor branch lines cutting, the real axis which is the path of integration. The standard formal 
asymptotic representation for this integral,

$$
F(x, k) \sim e^{i k x} \sum_{n=0}^{\infty}(l / k)^{n+1} f^{(n)}(x),
$$

can be obtained from equation (1) by repeated integration by parts. Further, any finite curtailment of this series can be shown to be a valid asymptotic representation in the sense of Poincaré. However the infinite series can converge only if $f$ is an entire function in the complex plane. For if $\sum f^{(n)}(x) k^{-n}$ be absolutely convergent for large enough values of $k, \sum f^{(n)}(x) z^{n} / n !$ must also be convergent, by the comparison test, since

$$
\operatorname{limit}_{n \rightarrow \infty}\left|f^{(n)}(x) z^{n} / n !\right| /\left|f^{(n)}(x) k^{-n}\right|=\operatorname{limit}_{n \rightarrow \infty}|k z|^{n} / n !<1
$$

for all finite $z$. Thus, for the class of functions under consideration in this paper, only a finite curtailment of the series in equation (2) can be used and then the question of estimating the remainder arises for functions $f$ which are other than polynomials. The discussion which follows cannot be applied when $f$ is free of finite singularities but has an essential singularity at infinity and what is appropriate in this case is not generally clear to the author.

The curtailed version, including the remainder, of the asymptotic representation (2) is

$$
F(x, k)=e^{i k x} \sum_{n=0}^{N-1}(l / k)^{n+1} f^{(n)}(x)+(l / k)^{N} \int_{x}^{\infty} e^{i k u} f^{N}(u) d u
$$

and the question under consideration is that of estimation of the remainder integral for values of $N$ large enough to make inappropriate, any further integration by parts. To do this for the general class of functions under consideration would excessively complicate the analysis and more convenient and illuminating results can be obtained from representations of each function $f$ in terms of its singularities. It will be seen that the singularities of $f$ control the errors in a quite simple way.

As a first stage it will be assumed that $f$ is holomorphic save for a finite number of poles and the analysis will be extended later to cover the case where $f$ has branch lines, and even an infinite number of poles, suitably arranged. Thus in the first stage $f$ can be replaced by the sum of its principal parts at each pole so that

$$
f=\sum_{n} \sum_{m} A_{n m}\left(u-u_{n}\right)^{-m}
$$

where both sums are finite. For this class of functions it suffices to consider the representative integral

$$
\int_{x}^{\infty} e^{i k u}\left(u-u_{0}\right)^{-m} d u
$$


Also a suitable choice of the origin of $x$ can be made so that $\mathscr{R} 1 u_{0}=0$ so that the behaviour of $F(x, k)$ is readily deducible from that of

$$
I(x, k)=\Gamma(m) \int_{x}^{\infty} e^{\imath k u} d u /(u-\lambda l)^{m}
$$

where $\lambda$ is real and non-zero. For this particular integral the result, corresponding to equation ( 3 ), is

$$
\begin{aligned}
I(x, k)= & -e^{i k x} \sum_{r=0}^{N-1} \Gamma(r+m)(l k)^{-(r+1)}\left(x-\lambda_{l}\right)^{-(r+m)} \\
& +(l k)^{-N} \Gamma(N+m) \int_{x}^{\infty} e^{\imath k u}(u-\lambda l)^{-(N+m)} d u .
\end{aligned}
$$

It does not seem appropriate to investigate the remainder integral in equation (5) in terms of two independently large parameters $N$ and $k$. An easier problem arises if one makes an appropriate choice of $N$, in terms of $k$, so that only a oneparameter estimate of the remainder has to be obtained. It should be remembered that representations of the type being sought are not unique so that there is a certain arbitrariness in the choice of procedure to be adopted. To the author it seems natural to proceed with the integration by parts until the stage is reached when its further application would lead to terms which are increasing in magnitude. This is merely a heuristic attempt to achieve efficiency in the arrangement of the representation but, since no approximation is involved, the result obtained would not be invalidated if the efficiency criterion is illusory. Since the ratio of the magnitudes of the successive terms in the series in equation (5) is $(r+m) /\left\{k\left|x-\lambda_{l}\right|\right\}$, the application of the efficiency arguments suggests choosing $N$ such that

$$
N+m=k\left\{x^{2}+\lambda^{2}\right\}^{\frac{1}{2}}+\delta
$$

where

$$
0 \leqq \delta<1
$$

For this choice of $N$ the remainder term can be written as

$$
\begin{aligned}
R_{N}(x) & =\Gamma(N+m)(\imath k)^{-N} \int_{x}^{\infty} e^{\imath k u}(u-\lambda l)^{-(N+m)} d u \\
& =\Gamma(N+m)(\imath k)^{-N} \int_{x}^{\infty} \exp \{\imath k u-(N+m) \ln (u-\lambda l)\} d u
\end{aligned}
$$

where, if $\lambda$ is positive the branch line joins $\lambda l$ to $\iota \infty$ and if $\lambda$ negative the branch line joins $\lambda_{l}$ to $-\imath \infty$. This arrangement of the integrand has produced a new form suitable for estimation by the method of steepest descents. There is a single saddle point at

$$
u-\lambda l=-l(N+m) / k \simeq-\imath\left(x^{2}+\lambda^{2}\right)^{\frac{1}{2}}
$$


which therefore lies on the imaginary axis in the lower half plane unless $x=0$ and $\lambda$ is positive when it may be at the origin. Also all paths of steepest descent from points on the real axis enter the upper half plane and eventually become asymptotically parallel to the imaginary axis. When $\lambda$ is negative these paths can be completed at infinity so as to reach real positive infinity without contribution from the completing arc. When $\lambda$ is positive however this is true only for those paths which reach imaginary infinity to the right of the branch line on the imaginary axis, namely those which originate from values $x$ which are non-negative. For negative values of $x$ the closure contribution to the remainder would involve extensive calculations if performed directly. The answer may be obtained more simply by noting that, for $x<0$, the original integral in equation (4) can be written as

$$
\int_{-\infty}^{\infty} e^{\imath k u} d u /(u-\lambda l)^{m}+\int_{x}^{-\infty} e^{\imath k u} d u /(u-\lambda l)^{m}
$$

The first integral can be evaluated exactly by applying the Residue Theorem in the upper half-plane and the result is a term smaller than and independent of any obtained in the asymptotic series. What is required is an estimate of the error for a given large value of the parameter. But in second integral the path of steepest descent now reaches infinity in a region from which there will be no completion contribution. If $L$ is used to denote the path of steepest descent from $x$ to positive imaginary infinity then it follows that for $x \geqq 0$ or $x<0, \lambda<0$

$$
R_{N}(x)=\Gamma(N+m)(\imath k)^{-N} \int_{L} \exp \{\imath k u-(N+m) \ln (u-\lambda l)\} d u
$$

whilst for $\lambda>0, x<0$

$$
\begin{aligned}
R_{N}(x)= & 2 \pi l(\imath k)^{m-1} e^{-k \lambda}+\Gamma(N+m)(\imath k)^{-N} \\
& \times \int_{L} \exp \{\imath k u-(N+m) \ln (u-\lambda l)\} d u .
\end{aligned}
$$

When the contribution from the path of steepest descent is to be estimated, two cases must be recognized. The integral is dominated in all cases by the contribution from near the terminal point $x$ but two cases arise according to whether this terminal is or is not near the saddle point. An examination of the Taylor series expansion of $\{\imath k u-(N+m) \ln (u-\lambda l)\}$ about $u=x$ reveals that the linear terms will predominate unless $\lambda$ is positive and $N^{-1} x^{-2}$ is not small. Thus when $\lambda$ is positive the case when $|x|$ is $0\left(N^{-\frac{1}{2}}\right)$ needs special consideration. In both cases there is no particular difficulty, other than the rather laborious nature of the calculations, preventing a number of terms in the asymptotic series for $R_{N}$ from being obtained. For the present investigation no attempt will be made to go beyond the dominant terms which may be shown to be: 
for $\lambda<0$ all $x$, or $\lambda>0 x \gg N^{-\frac{1}{2}}$

$$
\begin{aligned}
& R_{N} \sim \Gamma(N+m)(\imath k)^{-N}(x-\lambda l)^{-(N+m)} e^{\imath k x} /[l k-(N+m) /(x-\lambda l)], \\
& \text { for } \lambda>0,-x \gg N^{-\frac{1}{2}} \\
& \begin{aligned}
R_{N}(x) \sim & 2 \pi l(l k)^{m-1} e^{-k \lambda} \\
& -\Gamma(N+m)(\imath k)^{-N}(x-\lambda l)^{-(N+m)} e^{\imath k x} /[l k-(N+m) /(x-\lambda l)]
\end{aligned}
\end{aligned}
$$

and for $\lambda>0, x=O\left(N^{-\frac{1}{2}}\right)$

$R_{N}(x) \sim(2 \pi)^{\frac{1}{2}} l(l k)^{m-1} e^{-k \lambda} \int_{\lambda^{-\frac{1}{2}} k^{\frac{1}{2}} x}^{\infty} e^{-\frac{1}{2} \sigma^{2}} d \sigma^{1}$

which is seen to join smoothly the results for $x$ positive and negative. A careful application of Watson's lemma would permit the demonstration of the asymptotic nature of these results. Further it might be noted that there is nothing in principle preventing the determination of a complete asymptotic series representation for $R_{N}$ in each of the various cases. Moreover in principle the methods to be considered in later sections could be applied to derive the transcendentally small errors involved in replacing $R_{N}$ by its asymptotic representation.

Here however such calculations will not be carried out but rather the results obtained above will be examined for their implications for the more general asymptotic representation in equation (3). Thus corresponding to each pole of $f(u)$, say at $a+\imath b$, there will be a normal asymptotic series which should be curtailed at a value of $N$ approximately equal to $k\left\{(x-a)^{2}+b^{2}\right\}^{\frac{1}{2}}$ thereafter the remainder is more accurately represented, not by a continuation of the series, but by the form of a product of $\exp -k\left\{(x-a)^{2}+b^{2}\right\}^{\frac{1}{2}}$ times an asymptotic series in inverse powers of $k$ or a slightly more complicated form near $x=a$. In addition for certain relationships between the pole, the path and the terminal of integration which are easily determined by inspection there will be error terms of order $k^{m-1} \exp -k|b|$ which can be determined exactly. In the light of these results it is possible to state that the transcendentally small errors are of two types - (1) those dependent on the distance of the terminal point from the pole and (2) those dependent on the distance of closest approach of the path of integration to the pole, provided that it lies to the left. Errors of type I are clearly indicated by the fact that after a certain value of $N$ the terms in the asymptotic series increase in size so that they do not lead to hidden errors. Errors arising from the second source however are not so obliging so that they must be clearly recognized analytically and the asymptotic series adjusted accordingly. It is fortunate that the errors of this type are the easiest to compute.

1 In writing this result use has been made of the Stirling approximation to the $\Gamma$ function in order to more clearly demonstrate the smoothness of the join of the results. In applying extensions of this work for moderate values of $k$ such expansions of the factorial function might be inappropriate. 
It should be further noted that when the asymptotic series of equation (2) are being used for numerical purposes for moderate values of $k$ there may be considerable value in extracting from $f$ the contributions from the poles nearest to the terminal and treating these by extension of the methods described above. Such processes may extend considerably the range of values of $k$ over which asymptotic representations can be used to obtain accurate approximations. Moreover the above results give a very quick and easy method to decide the range of $k$ over which the asymptotic series will give answers of a given accuracy. All that is required is the location of the singularities of the function $f$ and the calculation of the error estimates from the dominant estimates obtained above.

The derivation of these results has been based on the assumption that $f$ has a finite number of poles and is without branch lines but these restrictions can be removed at least under a variety of useful assumptions. Thus if $f$ has in addition a finite number of branch lines across which the discontinuity is finite ${ }^{2}$, then the function $f$ can be represented in the form

$$
f(u)=\sum_{n, m} A_{n m}\left(u-u_{n}\right)^{-m}+\sum_{j}(2 \pi t)^{-1} \int_{L_{j}} \frac{\varphi_{j}(t)}{t-u} d t
$$

where $L_{j}$ denotes a branch line, $\varphi_{j}$ the discontinuity in $f$ across this branch line. This representation is valid provided that $\varphi_{j}$ satisfies a Hölder condition on the open line and appropriate conditions near the branch points. The integrals are only to be interpreted for $u$ not on $L_{j}$ but this restriction is of no importance here because the representation will be used only on contours which are completely disjoint from the $L_{j}$. Thus, when considering the contribution from any branch line to the integral in equation (1), it is valid to interchange the order of integration so that the typical contribution from any one branch line is

$$
(2 \pi l)^{-1} \int_{L_{j}} \varphi_{j}(t) d t \int_{x}^{\infty} e^{\imath k u}(t-u)^{-1} d u d t .
$$

The inner integral is of the type considered above and so the appropriate result from that theory can be inserted for the inner integral, the exact form depending on the geometrical relationship between the singularity and the path of integration. Whatever the relationship the inner integral will have a behaviour predominantly described by $\exp -k h(t)$ where $h(t)$ is a monotonic increasing function of the distance of $A$ from the branch point closest to the contour. The outer integral can thus be evaluated by standard asymptotic methods. In particular the results will be simplest when the branch line lies above and to the right of the point $x$. For such cases the contribution from such branch-lines merely has to be added to the formal result given by equation (2). In other cases there is some complication arising from the fact that the curtailment value of $N$ will be a function of $t$. Methods

2 This condition appears capable of relaxation in the light of subsequent analysis. 
for dealing with such examples will be discussed in section 4 through a particular example.

It would appear plausible, but no proof is offered, that the assumption of a finite number of poles and branch lines may be relaxed. Each pole or branch point gives to a series dominated by either $\exp \left\{-k\left[\left(x-a_{\imath}\right)^{2}+b_{1}^{2}\right]^{\frac{1}{2}}\right\}$ or $\exp -k\left|b_{1}\right|$ and it is therefore interesting to speculate that the formal answer will therefore have meaning provided these terms can be arranged in a sequence of non-decreasing distance without a finite limit point. It is tempting to regard such series as providing suitable asymptotic representations of the integrals. If this is so then the results obtained may well apply to entire functions like $p^{-1} \tanh p$.

\section{The simplest saddle point}

Here the simplest case of the analysis of a saddle point will be investigated. Integrals will be considered for which $g(u)$ is $-u^{2}$ and again only those functions $f$, which can be decomposed into their singularities, will be investigated. Thus the typical integral of interest, at least for the infinite range, is

$$
G(k)=\int_{-\infty}^{\infty} e^{-k u^{2}} d u /(u-\gamma)
$$

where $\gamma$ is a complex constant whose imaginary part is non-zero. The large parameter $k$ will again be assumed real although this restriction is not necessary. There are many modifications of standard methods available by which the asymptotic representation of $G(k)$ may be obtained and the following has been chosen because, although it is far from the most direct method of obtaining the asymptotic series, it does provide a very suitable form for the error. The above integral can be expressed in terms of the error integral ${ }^{3}$, as follows.

$$
\begin{aligned}
G(k) & =\int_{-\infty}^{0} e^{-k u^{2}} d u /(u-\gamma)+\int_{0}^{\infty} e^{-k u^{2}} d u /(u-\gamma) \\
& =2 \gamma \int_{0}^{\infty} e^{-k u^{2}} d u /\left(u^{2}-\gamma^{2}\right) \\
& =2 \gamma \int_{0}^{\infty} e^{-k u^{2}} d u /\left(u^{2}-\alpha^{2}\right)
\end{aligned}
$$

where $\alpha$ is that square root of $\gamma^{2}$ which lies in the upper half-plane. The integral $G(k)$, regarded as a function of $\alpha$ is holomorphic in the upper half $\alpha$-plane but in the sector, $\pi / 4<\varphi=\arg \alpha<3 \pi / 4$, the following manipulations are valid:

${ }^{3}$ A suggestion for which the author is grateful to Dr. P. B. Chapman. 


$$
\begin{aligned}
\int_{0}^{\infty} e^{-k u^{2}} d u /\left(u^{2}-\alpha^{2}\right) & =\int_{0}^{\infty} e^{t(\varphi-\pi / 2)} e^{-k u^{2}} d u /\left(u^{2}-\alpha^{2}\right) \\
& =\frac{l}{\alpha} \int_{0}^{\infty} e^{k x^{2} v^{2} d v /\left(v^{2}+1\right)},\left(u=v \alpha e^{-\imath \pi / 2)}\right. \\
& =\frac{l}{\alpha} \int_{0}^{\infty} e^{-z v^{2} d v /\left(v^{2}+1\right)},\left(z=-k \alpha^{2}, \mathscr{R} l z>0\right) \\
& =\frac{l}{2 \alpha} \int_{0}^{\infty} e^{-z t} d t t^{-\frac{1}{2}}(1+t)^{-1} \cdot\left(t=v^{2}\right) \\
& =\{l /(2 \alpha)\} \Gamma\left(\frac{1}{2}\right) e^{z} \operatorname{erfc} z^{\frac{1}{2}},
\end{aligned}
$$

where the $z$-plane is cut along the negative real axis, which result follows from the identification of the integral with the confluent hypergeometric function and the complete error integral (see Erdelyi (3)). The analytic continuation $\{l /(2 \alpha)\} \Gamma\left(\frac{1}{2}\right) \exp \left(-k \alpha^{2}\right) \operatorname{erfc}\left(-k \alpha^{2}\right)^{\frac{1}{2}}$, which is holomorphic in the upper half $\alpha$-plane, is thus the value of the integral in this upper half-plane.

For the present purposes it is convenient to derive the asymptotic representation from the integral formula

$$
\operatorname{erfc}\left(-k \alpha^{2}\right)^{\frac{1}{2}}=\int_{\left(-k \alpha^{2}\right)^{\frac{1}{2}}}^{\infty} \exp \left(-t^{2}\right) d t
$$

and its asymptotic representation. In deriving such representations, two cases must be recognized, dependent on the sign of $\mathscr{R} l\left\{-k \alpha^{2}\right\}^{\frac{1}{2}}$. When this is nonnegative the path of steepest descent goes to $+\infty$. But when it is negative, it goes to $-\infty$, so that one first writes

$$
\begin{aligned}
\operatorname{erfc}\left(-k \alpha^{2}\right)^{\frac{t}{2}} & =\int_{-\infty}^{\infty} \exp \left(-t^{2}\right) d t+\int_{\left(-k \alpha^{2}\right)^{\frac{1}{2}}}^{\infty} \exp \left(-t^{2}\right) d t \\
& =\pi^{\frac{1}{2}}-\int_{-\left(-k \alpha^{2}\right)^{\frac{1}{2}}}^{\infty} \exp \left(-t^{2}\right) d t
\end{aligned}
$$

and then this integral may be treated as when $\mathscr{R} l\left\{-k \alpha^{2}\right\}^{\frac{1}{2}}$ is non-negative. It thus follows that in the sectors $0<\arg \alpha<\pi / 4$ and $3 \pi / 4<\arg \alpha<\pi$, there are additional terms in the asymptotic representation of $G(k)$ which were exponentially small and whose presence is not necessarily indicated by the numerical behaviour of the asymptotic series.

The above analysis indicates that the complete asymptotic representation of $G(k)$ can be deduced once the,corresponding type representation is known for

$$
\operatorname{erfc} \omega=\int_{\omega}^{\infty} \exp \left(-t^{2}\right) d t
$$

when $|\omega|$ is large and $\mathscr{R} l \omega \geqq 0$. If only the conventional type asymptotic 
representation is required the standard representation of erfc $\omega$ for real large positive $\omega$ provides the series. If however error estimates are required other methods may be more useful. The forms of error that may arise can be deduced most readily from the results of section 2 by transforming the error integral into an integral closely analogous to that considered in that section. This may be achieved as follows, where the appropriate transformation is indicated after each equation,

$$
\begin{gathered}
\int_{\omega}^{\infty} \exp \left(-t^{2}\right) d t=\exp \left(-\omega^{2}\right) \int_{0}^{\infty} \exp \left\{-2 \omega u-u^{2}\right\} d u \quad(t=\omega+u) \\
=\frac{1}{2} \exp \left(-\omega^{2}\right) \int_{0}^{\infty} \exp (-v) d v\left(v+\omega^{2}\right)^{-\frac{1}{2}} \quad\left(v=2 u \omega+u^{2}\right) \\
=\frac{1}{2} \exp \left(-\omega^{2}\right) \int_{0}^{i \infty} \exp (-v) d v\left(v+\omega^{2}\right)^{-\frac{1}{2}} \text { (by deforming contour) } \\
=\frac{1}{2} \exp \left(-\omega^{2}+t \pi / 4\right) \int_{0}^{\infty} \exp (-\imath t) d t\left(t-\omega^{2} \imath\right)^{-\frac{1}{2}} \quad(v=t t) \\
=\frac{1}{2}|\omega| \exp \left(-\omega^{2}+\imath \pi / 4\right) \mid \int_{0}^{\infty} \exp \left(-\imath u|\omega|^{2}\right) d u\left(u-\sigma^{2} \imath\right)^{-\frac{1}{2}}(\sigma=\omega /|\omega|)
\end{gathered}
$$

The factor $(-1)$ inside the exponential in the integrand changes the locations of the branch points which call for special consideration but the essentials of the analysis are those of section 2 . Thus when the path of steepest descent, now in the lower half plane, passes near the branch-line erfe terms will occur which will effect the smooth transition between the cases where there is a term $\pi^{\frac{1}{2}}$ and when there is not. In all cases the techniques considered in section 2 could be applied but a rather more direct route is available for the function with the specific branch line in the above integral.

Thus if one works with the second of the above equations, namely

$$
\operatorname{erfc} \omega=\frac{1}{2} e^{-\omega^{2}} \int_{0}^{\infty} e^{-v} d v\left(v^{1}+\omega^{2}\right)^{-\frac{1}{2}},
$$

and applies the standard integration by parts technique, one obtains

$$
\begin{aligned}
2 e^{\left(\omega^{2}\right.} \operatorname{erfc} \omega= & \sum_{r=0}^{N-1} \frac{(2 r-1) !}{(r-1) !} 2^{-(2 r-1)} \omega^{-(2 r+1)}+\frac{(2 N-1) !}{(N-1) !} 2^{-(2 N-1)} \\
& \times \int_{0}^{\infty} e^{-v}\left(v+\omega^{2}\right)^{-(2 N+1) / 2} d v
\end{aligned}
$$

A straightforward investigation reveals that the last term in the series will not be the smallest if $N\left(N-\frac{1}{2}\right)(N-1)^{-1}$ exceeds $|\omega|^{2}$ so that, as before, $N$ is chosen as the greatest integer for which this is not true and then one seeks an alternative 
method of estimating the error integral. Thus one writes the remainder integral in the form

$$
\int_{0}^{\infty} e^{-v}\left(v+\omega^{2}\right)^{-(2 N+1) / 2} d v=\int_{0}^{\infty} \exp \left\{-v-\frac{1}{2}(2 N+1) \ln \left(v+\omega^{2}\right)\right\} d v .
$$

where the branch line is chosen so as not to cut the real axis. If $\mathscr{R l} \omega^{2}$ is nonnegative, increasing the size of $N$ merely displaces the saddle point further to the left and the saddle point therefore does not contribute the dominant term in the integral. This is the case which arises in the next section, and since this paper is not intended to provide complete results, only that case will be considered here. Then the integral is dominated by its behaviour in the neighbourhood of the origin but it is not appropriate to use the same exponential form as previously. If the argument of the exponential is replaced by the early terms in its Taylor series by writing

$$
\begin{aligned}
v+\frac{1}{2}(2 N+1) \ln \left(v+\omega^{2}\right)= & \frac{1}{2}(2 N+1) \ln \omega^{2}+v\left\{1+\frac{1}{2}(2 N+1) \omega^{-2}\right\} \\
& -\frac{1}{4}(2 N+1) \omega^{-4} v^{2}+O\left(N \omega^{-6} v^{3}\right)
\end{aligned}
$$

and use is made of the fact that $N$ is $O\left(|\omega|^{2}\right)$, it is apparent that the integral is dominated by the first two terms in this series and that the additional terms contribute only small corrections which can be calculated by standard means. Thus

$$
\begin{aligned}
\int_{0}^{\infty} e^{-v}\left(v+\omega^{2}\right)^{-(2 N+1) / 2} d v & =\omega^{-(2 N+1)} \int_{0}^{\infty} e^{-v\left\{1+\frac{1}{2}(2 N+1) \omega^{-2}\right\}} d v\left\{1+O\left(|\omega|^{-2}\right\}\right. \\
& =\omega^{-(2 N+1)}\left\{1+\frac{1}{2}(2 N+1) \omega^{-2}\right\}^{-1}\left\{1+O\left(|\omega|^{-2}\right)\right\}
\end{aligned}
$$

It thus follows by combining these results that,

$$
\begin{aligned}
G(k) & =1 /(4 l) \Gamma\left(\frac{1}{2}\right)\left\{\sum_{r=0}^{N-1} \frac{(2 r-1) !}{(r-1) !} 2^{-(2 r-1)}\left(-k \gamma^{2}\right)^{-(2 r+1) / 2}\right. \\
& +\frac{(2 N-1) !}{(N-1) !} 2^{-(2 N-1)}\left(-k \gamma^{2}\right)^{-(2 N+1) / 2}\left[1-\frac{1}{2}(2 N+1)\left(k \gamma^{2}\right)^{-1}\right]^{-1}\left\{1+O\left(k^{-1}\right)\right\}
\end{aligned}
$$

for $5 \pi / 4<\arg \gamma \simeq 7 \pi / 4$.

If it were so desired the factorial functions could be replaced by their Stirling formula approximations. However in cases where it is desired to apply these results for only moderate values of $k$ this might be inadvisable. Such a replacement gives the estimate $O\left(N^{-1} \exp -N\right)$ for the remainder term.

\section{A more general saddle point}

The analysis in this section has been undertaken with the view of clarifying the factors which control the orders of the errors when asymptotic representations of integrals of the form 


$$
\int_{a}^{b} \exp [k g(u)] f(u) d u
$$

are obtained by saddle point methods. The results of the previous two sections indicate clearly the importance of the singularities of $f(u)$ and their distance from the end-points, the path (for certain geometries) and the saddle point in section (3). Here a simple example will be considered in order to obtain a guide as to how the properties of $g(u)$, assumed to be entire, may affect the errors in the asymptotic representations. With this aim in view, it seems appropriate to choose the simplest possible $f(u)$, namely unity, and to choose a $g(u)$ of the simplest form which is not essentially equivalent to the linear and quadratic forms considered in the previous sections. A cubic form for $g(u)$ leads to integrals related to the Airy Functions and the particular integral chosen is the one taking exponentially small values in the range of values of $k$ of interest so that the answers obtained will not be those obtained more simply by deforming the path of integration.

Thus consider the integral

$$
I(k)=\int_{L} \exp k\left(u-\frac{1}{3} u^{3}\right) d u
$$

where $L$ is a simple arc joining $\infty \exp (4 \pi l / 3)$ to $\infty \exp (2 \pi l / 3)$ so that this integral can be expressed as $2 \pi \imath k^{\frac{1}{3}} A l\left(k^{\frac{3}{3}}\right)$. The standard asymptotic representation of this function can be obtained by deforming the path of integration to pass through the saddle point at $u=-1$ and making appropriate series expansions about that point. This leads to an exponential function of argument $(-2 k / 3)$ times an asymptotic series in negative fractional powers of $k$. Here interest will centre on the nature of the error if the asymptotic series is curtailed at its smallest term and the problems involved if additional terms are then sought which lead to accurate answers for smaller values of $k$ than those for which the normal asymptotic series may be used.

The integral may be transformed to the type considered in the previous section by means of the mapping defined by

$$
-\xi^{2}=\frac{2}{3}+u-\frac{1}{3} u^{3}
$$

This mapping has been chosen so that the saddle point at $u=-1$ coincides with the origin in the $\xi$-plane and with an appropriate choice of the square root for $\xi$ the mapping of a neighbourhood of $u=1$ onto a neighbourhood of $\xi=0$ is one-one. Further, let the branch be chosen so that the sense of direction of $L$ is mapped onto the sense of direction of the real line in the positive direction. The global mapping is not one - one without the introduction of an additional branch line which originates at the other zero of the derivative of $u-\left(\frac{1}{3}\right) u^{3}$. It is apparent that for general $g(u)$ there will be branch lines associated with every other saddlepoint other than the one being used for the method of steepest descents so 
that the present example does serve to indicate the necessary lines of development for more general functions $g(u)$. For the simple case of equation $(10)$, where there is only one other saddle-point, namely that at $u=1$, the region to the left of the curve $\operatorname{Im}\left\{u-\frac{1}{3} u^{3}-\frac{2}{3}\right\}=0$ will be mapped, in a one-one manner, onto the $\xi$-plane cut from $\xi=-\left(\frac{4}{3}\right)^{\frac{1}{2}} \imath$ to $-\imath \infty$.

It follows that equation (10) may be rewritten therefore in the form

$$
I(k) e^{\frac{2}{3} k}=\int_{-\infty}^{\infty} e^{-k \xi^{2}} f(\xi) d \xi
$$

where

$$
f(\xi)=\frac{d u}{d \xi}=-2 \xi /\left(1-u^{2}\right)
$$

Because of the one-one mapping of the neighbourhood of $u=-1$ onto $\xi=0$ the only singularities of $f(\xi)$ arise from the discontinuity in $f(\xi)$ across the branch line on the negative imaginary axis. Further an explicit determination of this discontinuity could be derived from an examination of the relevant roots of Cardan's solution of the cubic equation (11) for $u$ in terms of $\xi$. In more general cases the evaluation of the discontinuity in $f(\xi)$ would need numerical evaluation. Of greater importance here is the fact that equation (12) is of the form of integrals considered in section 3 so that it is possible to say, almost immediately, something about the minimum error which can be achieved using the conventional asymptotic representation

$$
I(k) e^{2 k / 3} \sim(2 \pi)^{\frac{1}{2}} \sum_{r=0}^{\infty} f^{(2 r)}(0)\left\{2^{2 r} r ! k^{(2 r+1)}\right\}^{-1} .
$$

For $f(\xi)$ admits of the integral representation

$$
f(\xi)=(2 \pi i)^{-1} \int_{\left(\frac{4}{3}\right) \frac{1}{2}}^{\infty} \frac{\varphi(t)}{\xi+t t} d t
$$

where $\varphi$ is related to the discontinuity in $f$ across the branch line. Thus equation (12) may be rewritten as

$$
I(k) e^{2 k / 3}=(2 \pi l)^{-1} \int_{\left(\frac{k}{3}\right)^{\frac{1}{2}}}^{\infty} \varphi(t) \int_{-\infty}^{\infty} e^{-k \xi^{2}}(\xi+l t)^{-1} d \xi d t .
$$

where the inversion of the order of integration is justified. Direct application of the procedures of section (3) to the inner integral would produce some difficulty, because the value of $N$ would depend upon $t$, so that the order of summation and integration with respect to $t$ would not be easily interchanged. It is therefore convenient to choose $N$ as the largest integer such that $N\left(N-\frac{1}{2}\right)(N-1)^{-1}$ does not exceed $4 k / 3$. With $N$ so chosen it follows from the analysis of the previous section that 


$$
\begin{aligned}
I(k) e^{2 k / 3}= & (2 \pi l)^{-1} \int_{\left(\frac{4}{3}\right)^{\frac{1}{2}}}^{\infty} \varphi(t)(2 l)^{-1} \Gamma\left(\frac{1}{2}\right) \\
& \times \int_{r=0}^{N-1} \frac{(2 r-1) !}{(r-1) !} 2^{-(2 r-1)}\left(k t^{2}\right)^{-(2 r+1) / 2} \\
& \left.+\frac{(2 N-1) !}{(N-1) !} 2^{-(2 N-1)} \int_{0}^{\infty} e^{-v}\left(v+k t^{2}\right)^{-(2 N+1) / 2} d v\right\} d t
\end{aligned}
$$

It follows from the extended Cauchy formula that

$$
\begin{aligned}
I(k) e^{2 k / 3}= & (2 \pi)^{\frac{1}{2}} \sum_{r=0}^{N-1} f^{(2 r)}(0)\left\{2^{2 r} r ! k^{(2 r+1) / 2}\right\}^{-1} \\
& -\frac{(2 N-1) !}{(N-1) !} \frac{1}{4 \pi} \Gamma\left(\frac{1}{2}\right) \int_{\left(\frac{4}{3}\right)^{\frac{1}{2}}}^{\infty} \varphi(t) \int_{0}^{\infty} e^{-v}\left(v+k t^{2}\right)^{-(2 N+1) / 2} d v d t .
\end{aligned}
$$

In the inner integral in the remainder term, one uses Taylor expansions to show

$$
\begin{gathered}
\exp -\left\{v+\frac{1}{2}(2 N+1) \ln \left(v+k t^{2}\right)\right\}=\left(k t^{2}\right)^{-(2 N+1) / 2} \exp \left\{v \left[1+\frac{1}{2}(2 N+1)\left(k t^{2}\right)^{-1}\right.\right. \\
\left.+O\left(N k^{-2} t^{-4}\right)\right\}
\end{gathered}
$$

so that, since $N$ is approximately $4 k / 3$, the method of steepest descents may be applied. The remainder term is therefore

$$
\begin{aligned}
-\frac{(2 N-1) !}{(N-1) !} \frac{1}{4 \pi} \Gamma\left(\frac{1}{2}\right) & \int_{\left(\frac{4}{3}\right)^{\frac{1}{2}}}^{\infty} \varphi(t) k^{-(2 N+1) / 2} \exp \{-(2 N+1) \ln t\} \\
& \times\left(1+\frac{4}{3} t^{-2}\right)^{-1} d t\left[1+O\left(k^{-1}\right)\right]
\end{aligned}
$$

This integral is dominated by the contribution from near $t=\left(\frac{4}{3}\right)^{\frac{1}{2}}$ and may be evaluated by relatively standard applications of the method of steepest descents when allowance is made for the fact that $\varphi(t)$ has a square root singularity at that point. It can also be shown, as at the end of section 3 , that the remainder term, apart from an algebraic factor, is $O\{\exp (-4 k / 3)\}$. It therefore follows that $I(k)$ admits of the asymptotic representation

$$
\begin{aligned}
I(k) \sim & e^{-2 k / 3}\left\{(2 \pi)^{\frac{1}{2}} \sum_{r=0}^{N-1} f^{(2 r)}(0)\left[2^{2 r} r ! k^{(2 r+1) / 2}\right]^{-1}\right. \\
& \left.+e^{-4 k / 3} k^{\delta} \sum_{r} c_{r} k^{-r}\right\}
\end{aligned}
$$

where $\delta$ and $c_{r}$ are constants which could be determined by standard, but laborious, methods. The last series is merely asymptotic but computationally it would not seem practicable to determine the appropriate point of curtailment and the asymptotic form of the further remainder by the methods of this paper. It might however be possible to determine its exponential order. 


\section{Conclusions}

Conventional methods for the determination of asymptotic representations for integrals of the form $\int_{a}^{b} f(u) \exp \{k g(u)\} d u$ yield contributions from the endpoints $a$ and $b$, any saddle points which must be used on paths of minimum ascent between the end-point and from any singularities of $f(u)$ which must be crossed in deforming the path of integration to one of steepest descent. The analysis of this paper, incomplete though it is, clearly indicates the factors which control the orders of the smallest errors which may be achieved by appropriately curtailing the asymptotic representations. It also provides a clear indication as to the optimum method of curtailing a given series. It has been shown, at least for a fairly broad class of functions $f$ and $g$, that the minimum errors are controlled by the singular points of the function $f(u)$ and the zeros of the derivative $g^{\prime}(u)$. In all the cases examined the magnitude of these minimum errors is governed by the distance between the singular point of $f(u)$ or the zero of $g^{\prime}(u)$ from either the end-points or the saddle-points about which conventional methods would lead one to make expansions. The exponential factor in the minimum error estimate can be written down simply in terms of the appropriate distance but more accurate estimates require more extensive calculation. The methods thereby provide relatively simple 'a priori' estimates of the range of $k$ for which the conventional asymptotic series will provide numerical estimates of a given accuracy, purely from a knowledge of the singularities of $f(u)$ and the zeros of $g^{\prime}(u)$.

Further there are cases when the source of biggest error is too close to the path of integration so that a potentially good asymptotic expansion is rendered relatively useless. In many such cases an extension of the methods of the present paper would permit the computation of extended expansions which would increase the useful domain of the expansions. It should be noted that this would require merely the discussion of a simpler form of function based on the awkward singularity or saddle point and the remainder of the contributions could be calculated by conventional means. There is the additional feature of the present analysis that is clearly indicates when certain terms, whose presence is not indicated by the numerical convergence of the standard asymptotic series, actually occur and how large their contribution will be. It thus provides a means of ensuring that asymptotic representations, apparently well behaved numerically, are not used if they lead to significant errors. It would appear to warrant further investigation as to whether these methods would provide means of obtaining better asymptotic representations near the boundaries of arg $k$ for which the standard representations become invalid. It appears to the author that results of the present form may well provide an answer to the serious limitations of present methods which occur in the cases such as discussed by Olver [2]. 


\section{Acknowledgments}

This work was done at the United States Army Mathematics Research Center, University of Wisconsin. The author gratefully acknowledge the hospitality of that institution and support under Contract No.: DA-31-124-ARO-D-462.

\section{References}

[1] J. J. Mahony, 'The reflection of short waves by a variable medium', Quart. App. Math. 25 (1967), 313.

[2] F. W. J. Olver, 'Error Bounds for Asymptotic Expansions', (an article in C. H. Wilcox, (Ed.) Asymptotic solutions of differential equations and their applications, Wiley, New York, 1964.)

[3] A. Erdelyi, (Ed.) Higher Transcendental Functions, Volume I (McGraw Hill, New York, 1953 , p. 266 and p. 255.)

Department of Mathematics

University of Western Australia 